\title{
ARTIGO DE REVISÃo Conhecimento e intervenção do cuidador na doença de Alzheimer: uma revisão da literatura
}

\author{
Knowledge and caregivers' intervention in \\ Alzheimer's disease: a literature review \\ Cinthya Dolores Santos Maia Leite', Terce Liana Mota de Menezes², Érica Verônica de Vasconcelos Lyra³, \\ Cláudia Marina Tavares de Araújo
}

\section{RESUMO}

Objetivo: Analisar de forma sistemática o conhecimento da doença de Alzheimer (DA) e as estratégias adotadas pelo cuidador para lidar com os sintomas apresentados pelo idoso com DA. Métodos: Levantamento de estudos publicados nas bases de dados PubMed, MedLine, Lilacs e SciELO, utilizando os seguintes descritores e termos livres para estratégia de busca, de acordo com suas definições no DeCS e no MeSH: "cuidadores" or "educação em saúde" or "treinamento" or "cuidadores de idosos" and "doença de Alzheimer"; "elderly caregiving" or "training" or "health education" or "caregivers" and "Alzheimer disease"; "cuidadores de personas mayores" or "formación" or "educación en salud" or "cuidadores" and "enfermedad de Alzheimer".

Resultados: Foram eleitos 12 artigos para análise e leitura na íntegra. Os artigos foram classificados em três categorias: 1) Conhecimento acerca do declínio cognitivo e funcional da doença de Alzheimer; 2) Conhecimento acerca dos sintomas psicológicos e comportamentais da demência (SPCD); 3) Conhecimento acerca do comprometimento da linguagem. Na maioria dos estudos, verificou-se que a orientação a respeito do que é a enfermidade e a sua evolução pode interferir na maneira como o cuidador planeja e executa as ações de cuidado.

\section{Palavras-chave}

Cuidadores, idosos, doença de Alzheimer, educação em saúde, padrão de cuidado.
Conclusão: É extremamente necessário criar oportunidades para que os cuidadores familiares e profissionais conheçam a doença para compreender o paciente e, consequentemente, as possibilidades de atuação (seguras e eficazes) nesse paciente. Isso é possível mediante implementação de programas psicoeducativos capazes de fornecer informações e orientações práticas para melhorar a assistência oferecida por cuidadores aos pacientes com DA.

\section{ABSTRACT}

Objective: To analyze systematically the knowledge of Alzheimer's disease (AD) and the strategies used by the caregiver to act on the symptoms presented by the elderly with AD. Methods: Search of articles at PubMed, MedLine, Lilacs and SciELO, using the following keywords and free terms to search strategy, according to their definitions in DeCS and MeSH: "cuidadores" or "educação em saúde" or "treinamento" or "cuidadores de idosos" and "doença de Alzheimer"; "elderly caregiving" or "training" or "health education" or "caregivers" and "Alzheimer disease"; "cuidadores de personas mayores" or "formación" or "educación en salud" or "cuida-

1 Universidade Federal de Pernambuco (UFPE), Programa de Pós-Graduação em Saúde da Comunicação Humana. 2 UFPE, Hospital das Clínicas, Ambulatório de Neurologia Cognitiva/Comportamental.

3 UFPE, Departamento de Terapia Ocupacional.

Recebido em

Aprovado em

$26 / 2 / 2014$
Endereço para correspondência: Cinthya Dolores Santos Maia Leite Rua José Bonifácio, 529/502, Torre 50710-001 - Recife, PE, Brasil

E-mail: cinthyaleite@casasaudavel.com.br 


\section{Keywords}

Caregivers, elderly, Alzheimer's disease, health education, standard of care. dores" and "enfermedad de Alzheimer". Results: We found 12 articles to analyze and reading in full. The articles were classified into three categories: 1) Knowledge about the cognitive and functional decline of Alzheimer's disease; 2) Knowledge about the behavioral and psychological symptoms of dementia (BPSD); 3) Knowledge about language impairment. In most studies, it was found that the orientation about the disease and its evolution can interfere with how the caregiver plans and executes the actions of care. Conclusion: It is extremely necessary to create opportunities for family caregivers and professionals know the disease to understand the patient and the possibilities for action (safe and effective) with him. It is possible through implementing psychoeducational programs that provide information and practical guidance to improve the care provided by caregivers of patients with AD.

\section{INTRODUÇÃO}

Desde a sua primeira descrição em 1907, por um grupo de psiquiatras alemães, até os dias atuais, a doença de Alzheimer (DA) continua desafiando o tirocínio científico. Por isso, ainda é rodeada de mitos, nos quais acreditam não apenas pacientes e cuidadores, mas também médicos e profissionais de saúde'. Tipo mais comum entre mais de 140 tipos de demência, a DA é crônica, neurodegenerativa e progressiva, além de não ter ainda causa definida².

O envelhecimento é um fator de risco para o desenvolvimento da enfermidade, visto que os idosos compreendem a faixa etária mais acometida por esse tipo de demência². A enfermidade atinge cerca de 25 milhões de pessoas em todo o mundo, e em torno de $90 \%$ dos casos têm início após os 65 anos de idade ${ }^{1,2}$. Isso significa que a DA mantém relação diretamente proporcional ao aumento da idade 3 .

O comprometimento da memória, especialmente para fatos recentes, é o primeiro sinal mais evidente da enfermidade. No entanto, ao longo da evolução da doença, outros sintomas também despontam, como prejuízo da linguagem e dificuldade para realizar as tarefas do dia a dia, mesmo as mais simples ${ }^{4}$. Por isso, o idoso com a enfermidade precisa de auxílio específico em seu cotidiano para manter a qualidade de vida ${ }^{5}$

Dessa maneira, é preciso considerar que, com o avançar da doença, as pessoas perdem a independência e a autonomia. Assim, faz-se necessário que o idoso com DA conte com o apoio de um cuidador nas atividades de vida diária. É ele quem deve ter responsabilidade pelos cuidados prestados ao idoso dependente. O cuidador pode ser um parente ou um profissional contratado pela família para exercer a função do cuidar, uma tarefa que exige preparo suficiente para lidar com o paciente que tem $\mathrm{DA}^{6}$.

O cuidador também precisa receber orientações da equipe médica e de outros profissionais de saúde, já que o cuidado é considerado uma intervenção não farmacológica importante e que deve ser associado ao tratamento medicamentoso ${ }^{7}$.

É importante ressaltar que só faz sentido cuidar de alguém quando este não consegue desempenhar suas ati- vidades de vida diária de maneira autônoma (ou seja, sem auxílio de outras pessoas), e frequentemente esses cuidados podem ir além do âmbito domiciliar, no sentido de satisfazer as necessidades básicas do sujeito8.

Para sentir-se seguro para lidar com as diversas manifestações no curso da DA, o cuidador precisa conhecer a enfermidade. Quando informado sobre a doença, ele possivelmente consegue construir uma convivência facilitada, porque pode se planejar e se reorganizar com mais segurança e melhor manejo diante da enfermidade ${ }^{5}$. Esse preparo ajuda o cuidador a lidar melhor com o idoso que tem DA e a sentir-se seguro para controlar os sintomas psicológicos e comportamentais da patologia, que podem incomodar até mais do que o próprio déficit de memória?.

Embora pesquisas confirmem que o cuidador bem informado é mais hábil na relação médico-paciente-família, o sistema de saúde ainda não legitima a atuação dele na intervenção na DA, pois oferta geralmente atendimentos isolados e em ações direcionadas somente ao doente. Assim, este estudo se justifica pela necessidade de o cuidador ter acesso a programas psicoeducativos que promovam a compreensão da história da doença em todo o seu curso e, consequentemente, o auxiliem a desenvolver estratégias adequadas no manejo e cuidados com o idoso portador de DA'.

As intervenções psicoeducacionais contribuem significativamente para a melhora do bem-estar do cuidador. Ainda é necessária, no entanto, uma padronização dessa abordagem em termos de estrutura, duração e conteúdos ministrados, a fim de que haja evidências mais precisas do efeito desse tipo de intervenção ${ }^{10}$.

Diante dessa realidade, realizou-se uma revisão de literatura com o objetivo de analisar o conhecimento desse tipo de demência e as estratégias adotadas pelo cuidador para lidar com os sintomas apresentados pelo idoso com DA.

\section{MÉTODOS}

Trata-se de uma revisão da literatura que permite a inclusão simultânea de pesquisas experimentais e não experimentais, a fim de se obter uma ampla compreensão do fenômeno 
em estudo. As etapas adaptadas para o desenvolvimento desta revisão foram: escolha do tema e do objeto de estudo, levantamento da questão da pesquisa ou definição do problema a ser investigado, escolha dos descritores e dos termos livres, levantamento dos artigos nas bases de dados, estabelecimento de critérios para inclusão e exclusão dos estudos, além da discussão dos resultados com a identificação de conclusões.

Para que os objetivos fossem atingidos, a seguinte questão norteadora foi delineada: como age o cuidador diante das necessidades, do comportamento e dos sinais apresentados pelos idosos com doença de Alzheimer?

Os critérios de inclusão utilizados foram: artigos originais que respondessem à questão norteadora, com resumos apresentados na base de dados. E os critérios de exclusão foram: editoriais, artigos de revisão da literatura e artigos que não respondessem à questão norteadora proposta por este estudo.

Para a busca dos descritores padronizados, lançou-se mão do MeSH (Medical Subjects Headings) e do DeCS (Descritores em Ciências de Saúde), utilizando-se como DeCS "doença de Alzheimer"; "cuidadores"; "educação em saúde", bem como seus equivalentes em espanhol e em inglês. Como MeSH, foram usados "Alzheimer disease"; "caregivers"; "health education"; "training". Recorremos também a termos livres, que são termos não encontrados no DeCS e MeSH, mas de relevância para a pesquisa. Em português, utilizamos os termos livres "cuidadores de idosos"; "treinamento". Em espanhol, os equivalentes foram "cuidadores de personas mayores"; "formación". Já em inglês, foi utilizado como termo livre "elderly caregiving".

Para otimização da pesquisa, combinações foram feitas entre descritores e termos livres. Assim, a estratégia específica para o cruzamento e a busca dos artigos utilizou as seguintes sintaxes: "cuidadores" or "educação em saúde" or "treinamento" or "cuidadores de idosos" and "doença de Alzheimer"; "elderly caregiving" or "training" or "health education" or "caregivers" and "Alzheimer disease"; "cuidadores de personas mayores" or "formación" or "educación en salud" or "cuidadores" and "enfermedad de Alzheimer".

A revisão da literatura foi realizada utilizando as bases de dados Medical Literature Analysis and Retrieval System Online (MedLine), Literatura Latino-Americana e do Caribe em Ciências da Saúde (Lilacs), Scientific Electronic Library Online (SciELO) e a base eletrônica ofertada pela US National Library of Medicine (PubMed). A busca de dados, realizada por dois pesquisadores de forma independente (seguindo os critérios de inclusão e exclusão), ocorreu entre agosto e outubro de 2012. As opiniões divergentes foram discutidas em reunião, momento em que foram feitos ajustes.

A seleção dos artigos para análise foi realizada primeiramente pela leitura dos títulos. Aqueles que não atenderam ao objetivo da pesquisa foram excluídos. Dos que ficaram, foi lido o resumo de todos os estudos encontrados na busca em cada uma das bases de dados. Foram descartados os artigos cujos resumos não entraram em consonância com a proposta desta revisão. O passo seguinte foi selecionar os artigos na íntegra e analisá-los. Realizou-se, ainda, a busca manual das listas de referências dos artigos incluídos na revisão.

\section{RESULTADOS}

A busca nas bases de dados estabelecidas resultou em 1.547 artigos. Depois de ser feita uma leitura sistematizada dos títulos e dos resumos, sempre respeitando os critérios de inclusão e de exclusão, foram eleitos 12 artigos para análise minuciosa. Detalhes do número de artigos encontrados e selecionados por cruzamento dos descritores estão esquematizados na figura 1.

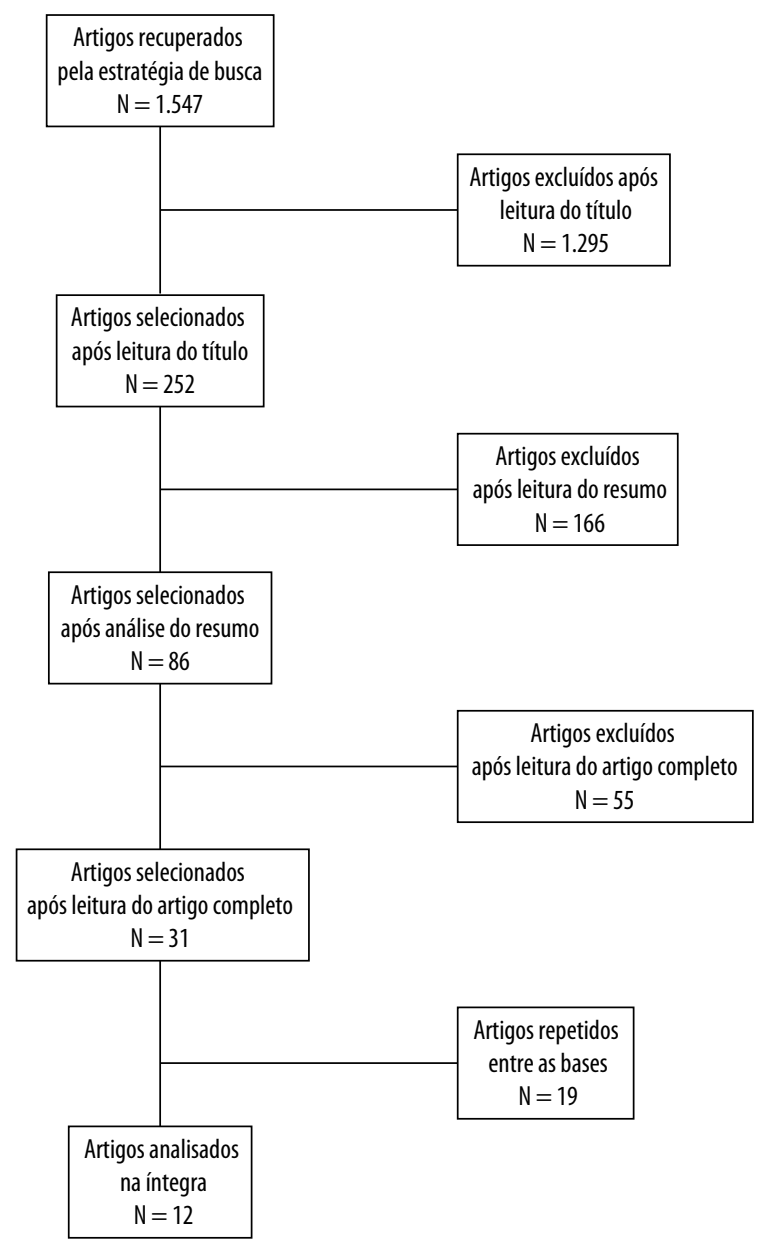

Figura 1. Fluxograma do número de artigos encontrados e selecionados após aplicação dos critérios de inclusão e exclusão, segundo descritores e base de dados.

Os 12 artigos selecionados foram organizados segundo ano, local, objetivo, amostragem, método utilizado e resultados. Detalhamento no quadro 1. 
Quadro 1. Estudos que revelam como o cuidador age e atua diante de um idoso com doença de Alzheimer (estão organizados segundo autor/ano, local, objetivo, amostragem, método e resultados principais)

\begin{tabular}{|c|c|c|c|c|c|}
\hline Autor/ano & Local do estudo & Objetivo & Amostragem & Método & Resultados principais \\
\hline $\begin{array}{l}\text { Amieva et al., } \\
2012\end{array}$ & França & $\begin{array}{l}\text { Explorar necessidades e } \\
\text { expectativas dos cuidadores } \\
\text { familiares de pessoas com DA } \\
\text { que solicitaram assistência } \\
\text { médica para seus parentes } \\
\text { com DA }\end{array}$ & $\begin{array}{l}645 \text { cuidadores de idosos } \\
\text { com DA em fase moderada }\end{array}$ & $\begin{array}{l}\text { Escala francesa de expectativas } \\
\text { do paciente para consulta médica, } \\
\text { completada por cuidadores durante a } \\
\text { visita médica }\end{array}$ & $\begin{array}{l}\text { Cuidadores relataram expectativas altas } \\
\text { ou muito altas diante das necessidades } \\
\text { relacionadas à informação sobre DA e } \\
\text { a habilidades de aprendizagem, a fim } \\
\text { de melhorar a gestão de vida diária do } \\
\text { parente }\end{array}$ \\
\hline $\begin{array}{l}\text { Navab et al., } \\
2012\end{array}$ & Irã & $\begin{array}{l}\text { Compreender experiências } \\
\text { de cuidadores familiares } \\
\text { iranianos de pessoas com DA }\end{array}$ & $\begin{array}{l}8 \text { cuidadores familiares } \\
\text { iranianos de pessoas } \\
\text { com DA }\end{array}$ & Entrevista semiestruturada & $\begin{array}{l}\text { Existe necessidade de proporcionar } \\
\text { educação continuada e apoio para } \\
\text { cuidadores familiares de pessoas com } \\
\text { DA. Enfermeiros estão entre profissionais } \\
\text { da linha de frente para apoiar e fornecer } \\
\text { informações para esses cuidadores }\end{array}$ \\
\hline $\begin{array}{l}\text { Yektatalab et } \\
\text { al., } 2012\end{array}$ & Irã & $\begin{array}{l}\text { Explorar percepções dos } \\
\text { cuidadores iranianos de } \\
\text { idosos com Alzheimer } \\
\text { nas instituições de longa } \\
\text { permanência de idosos }\end{array}$ & $\begin{array}{l}14 \text { cuidadores de idosos } \\
\text { com DA }\end{array}$ & Grupos focais e entrevistas & $\begin{array}{l}\text { Vários aspectos relacionados a cuidados } \\
\text { foram mencionados pelos participantes, } \\
\text { mas o principal foi o cuidado físico e } \\
\text { personalizado. Planos de educação } \\
\text { continuada para cuidadores podem } \\
\text { melhorar qualidade do atendimento e } \\
\text { qualidade de vida do paciente que vive } \\
\text { em instituição de longa permanência para } \\
\text { idosos }\end{array}$ \\
\hline $\begin{array}{l}\text { Williams, } \\
2011\end{array}$ & Estados Unidos & $\begin{array}{l}\text { Descrever o conhecimento } \\
\text { sobre comunicação na DA } \\
\text { dos cônjuges cuidadores e } \\
\text { determinar necessidades de } \\
\text { aprendizado sobre estratégias } \\
\text { de comunicação eficazes }\end{array}$ & $\begin{array}{l}16 \text { cuidadores } \\
\text { ( } 5 \text { homens e cinco } \\
\text { mulheres), com } 55 \text { anos ou } \\
\text { mais, casados com pessoas } \\
\text { portadoras de DA }\end{array}$ & $\begin{array}{l}\text { AD Communication } \\
\text { Knowledge Test, AD Communication } \\
\text { Problem Checklist, Self-Rated AD } \\
\text { Communication Scale }\end{array}$ & $\begin{array}{l}\text { Cônjuges cuidadores precisam entender } \\
\text { declínio da comunicação associado à DA. } \\
\text { Enfermeiros podem desempenhar papel } \\
\text { importante no apoio a casais, educando } \\
\text { os cuidadores sobre distúrbios de fala e } \\
\text { comunicação, bem como sobre estratégias } \\
\text { para vencê-los }\end{array}$ \\
\hline
\end{tabular}

$\begin{array}{ll}\begin{array}{l}\text { Ducharme et } \\ \text { al., } 2011\end{array} & \text { Canadá } \\ & \text { progtar eficácia de uma psicoeducacional } \\ & \text { individual concebido para } \\ & \text { facilitar a transição para } 0 \\ & \text { papel de cuidador após o } \\ & \text { diagnóstico de Alzheimer em } \\ & \text { um parente }\end{array}$
2010

Roque et al., Brasil 2009

Cisek et al., Polônia 2005 conhecimento e cuidado de cuidadores familiares de idosos portadores da doença de Alzheimer de orientação sobre estratégias comunicativas a cuidadores de idosos com DA moderada

Avaliar nível de desenvoltura da família para cuidar de
62 cuidadores do grupo de treinamento experimental e 49 do grupo de cuidados usuais for Caregiving scale, Revised Scale for Caregiving Self-Efficacy, Planning for Future Care Needs Scale, Knowledge of Services Scale, Carers' Assessment of Managing Index, 27-item Inventory of Socially Supportive Behaviors, The Family Caregiver Conflict Scale

14 cuidadores de idosos Entrevista semiestruturada com DA

Verificar eficácia de programa um parente com doença de Alzheimer
30 cuidadores de idosos com DA, sendo 19 mulheres e 11 homens
Questionário com itens que avaliam nível de conhecimento apresentado pelos membros da família, organização dos cuidados e formas de cuidar de uma pessoa com DA, escala e teste que reconhecem a adaptação da família ao parente com DA
Em comparação ao grupo de cuidados usuais, os cuidadores do grupo experimental estavam mais confiantes em lidar com situações de cuidado, perceberam estar preparados para prestar cuidados, foram mais capazes de planejar necessidades futuras de atenção de seu parente e fizeram uso mais frequente das estratégias de enfrentamento da resolução de problemas

Conhecimento dos cuidadores sobre DA limita-se a informações fornecidas pela mídia e pelo conhecimento do senso comum, o que contribui para cuidados pouco recomendáveis. Fornecer informações é fundamental para desenvolver no cuidador a tomada de decisão no momento de prestar cuidado ao idoso com DA

Depois do programa, verificaram-se: aumento na frequência de uso das estratégias e relato de outras mudanças relacionadas à comunicação. Houve eficácia do programa, uma vez que se verificaram mudanças no comportamento comunicativo dos cuidadores

0 nível de conhecimento sobre DA está associado ao nível de educação. Observouse que $67 \%$ das famílias cuidam do parente com DA sem pertencer a grupo de apoio. Para as famílias, adaptar-se à situação imposta pela DA é difícil, embora a maioria delas passe a dedicar parte da energia para o cuidado do paciente 
Quadro 1. Estudos que revelam como o cuidador age e atua diante de um idoso com doença de Alzheimer (estão organizados segundo autor/ano, local, objetivo, amostragem, método e resultados principais)

\begin{tabular}{|c|c|c|c|c|c|}
\hline Autor/ano & Local do estudo & Objetivo & Amostragem & Método & Resultados principais \\
\hline $\begin{array}{l}\text { Paton et al., } \\
2004\end{array}$ & $\begin{array}{l}\text { Inglaterra (Reino } \\
\text { Unido) }\end{array}$ & $\begin{array}{l}\text { Ter uma visão sobre a } \\
\text { compreensão dos cuidadores } \\
\text { diante das causas dos } \\
\text { sintomas comportamentais } \\
\text { dos idosos com DA }\end{array}$ & $\begin{array}{l}205 \text { cuidadores de idosos } \\
\text { com DA }\end{array}$ & Entrevista semiestruturada & $\begin{array}{l}\text { A maioria dos cuidadores atribui } \\
\text { sintomas cognitivos, comportamentais e } \\
\text { psicológicos da demência a causas que não } \\
\text { estão relacionadas à demência. Muitos } \\
\text { acreditam que a pessoa com demência tem } \\
\text { o controle sobre o seu comportamento e } \\
\text { que vai voltar ao normal }\end{array}$ \\
\hline $\begin{array}{l}\text { Caldeira e } \\
\text { Ribeiro, } 2004\end{array}$ & Brasil & $\begin{array}{l}\text { Identificar e analisar a } \\
\text { percepção que os cuidadores } \\
\text { de idosos têm sobre DA e os } \\
\text { cuidados prestados } \\
\text { a esses idosos }\end{array}$ & $\begin{array}{l}8 \text { cuidadores de idosos } \\
\text { com DA }\end{array}$ & Entrevista semiestruturada & $\begin{array}{l}\text { Apesar de os cuidadores não se sentirem } \\
\text { bem diante do cuidar, a maioria tem } \\
\text { interesse em aprender a cuidar melhor do } \\
\text { idoso e de si mesmo. Sugere-se assistência } \\
\text { de enfermagem sistematizada de educação } \\
\text { para os cuidadores de idosos com DA }\end{array}$ \\
\hline Werner, 2001 & Israel & $\begin{array}{l}\text { Avaliar nível de conhecimento } \\
\text { da família sobre DA }\end{array}$ & $\begin{array}{l}220 \text { cuidadores informais } \\
\text { de idosos com DA }\end{array}$ & $\begin{array}{l}\text { Questionário com } 17 \text { questões sobre DA } \\
\text { baseado no Alzheimer's } \\
\text { Disease Knowledge Test (ADK) }\end{array}$ & $\begin{array}{l}\text { Baixos níveis de conhecimento foram } \\
\text { encontrados, especialmente em itens } \\
\text { relacionados a prevalência, a causas e a } \\
\text { sintomas da doença. Mais pesquisas sobre } \\
\text { o conhecimento dos cuidadores sobre DA } \\
\text { devem ser promovidas, a fim de permitir } \\
\text { melhor tomada de decisões dos cuidadores } \\
\text { e melhor adequação às necessidades deles } \\
\text { para implementação de programas de } \\
\text { intervenção }\end{array}$ \\
\hline $\begin{array}{l}\text { Robinson et } \\
\text { al., } 2001\end{array}$ & Estados Unidos & $\begin{array}{l}\text { Examinar relações entre } \\
\text { distúrbios de comportamento } \\
\text { e impacto no cuidado em } \\
\text { cuidadores }\end{array}$ & $\begin{array}{l}30 \text { cuidadores de idosos } \\
\text { com DA }\end{array}$ & $\begin{array}{l}\text { Revised Memory and Behaviour Problem } \\
\text { Checklist; Cost of Care Index }\end{array}$ & $\begin{array}{l}\text { Cuidador não se sente à vontade diante } \\
\text { dos problemas de comportamento dos } \\
\text { idosos com DA. Essas descobertas têm } \\
\text { implicações para implementação de } \\
\text { programas de intervenção. Cuidadores } \\
\text { precisam receber formação específica para } \\
\text { entender e gerenciar o comportamento e a } \\
\text { depressão em pessoas com DA }\end{array}$ \\
\hline
\end{tabular}

A heterogeneidade dos artigos não permitiu a aplicação de tratamento estatístico (metanálise). Constatou-se que $75 \%$ (9) eram provenientes de publicações estrangeiras e $25 \%$ (3), nacionais. Entre as diversas diferenças entre os estudos, ressalta-se o número de indivíduos utilizados nos 12 artigos selecionados. Foram identificados desde estudos realizados com oito participantes ${ }^{11}$ até amostra representada por 645 entrevistados ${ }^{12}$.

As amostras maiores foram usadas para atender aos objetivos dos estudos com métodos quantitativos. Já as amostras menores foram das pesquisas qualitativas, cuja significância não se encontra na quantidade final de seus elementos, mas na maneira como se concebe a representatividade desses elementos e na qualidade das informações obtidas deles. Em estudos qualitativos, a seleção dos elementos decorre, sobretudo, da preocupação de que a amostra contenha e espelhe certas dimensões do contexto estudado ${ }^{13}$.

Cerca de 50\% dos manuscritos apoiam-se em instrumentos quantitativos ${ }^{12,14-18}$, o que inclui escalas, checklists e questionários fechados. São pesquisas cujo objetivo se volta a quantificar as expectativas dos cuidadores e os níveis de conhecimento e a avaliar a desenvoltura deles diante do idoso com Alzheimer. Os outros 50\% dos artigos têm essên- cia naturalmente qualitativa ${ }^{4,6,11,19-21}$ e recorrem a entrevistas semiestruturadas, a grupos focais e a questionários abertos. Esses exploram, em geral, percepções dos cuidadores sobre a doença e têm como meta compreender a experiência desses profissionais, o que não dá para ser observado por meio de instrumentos quantitativos.

\section{DISCUSSÃO}

Nos estudos analisados para esta revisão, percebe-se a necessidade de planejamento do cuidado, de organização da logística para execução do cuidar e de educação dos cuidadores.

É importante frisar que todos os estudos brasileiros selecionados ${ }^{4,6,21}$ foram feitos com base em métodos qualitativos, o que sugere uma tendência dos pesquisadores nacionais a se interessarem por instrumentos teóricos que sirvam de aporte para se compreender e interpretar a realidade quando se trata de analisar a experiência dos cuidadores com as ações de cuidados prestados ao idoso com Alzheimer.

Em relação aos anos de publicação dos artigos, compreendidos entre 2001 e 2012, houve dois artigos em 2001, dois em 
2004, um em 2005, um em 2009, um em 2010, dois em 2011 e três em 2012. Pode-se observar que foi realmente a partir da primeira década do século XXI que houve maior disseminação de conhecimentos sobre DA. Isso tem levado os especialistas das áreas da neurologia, da psiquiatria, da geriatria e da enfermagem a buscarem atualização nessa área.

A intensificação na produção de manuscritos sobre o tema, evidenciada na primeira década deste século, comprova que a DA migrou de um extremo a outro quando saiu de uma condição em que era considerada doença rara (até quase a década de 1970) para se transformar em uma epidemia dos anos 2000. Para retratar a evolução do número de publicações sobre a doença ao longo de décadas, Caixeta' constatou apenas 18 artigos na década de 1960, em levantamento de publicações sobre a doença e indexados no PubMed. Nos anos de 2000 a 2010, esse número chega a 52.045.

Outro ponto que deve ser levado em consideração, na análise dos resultados aqui apresentados, é o percentual (25\%) de artigos brasileiros selecionados. Isso espelha o fato de que a comunidade acadêmica brasileira tem se esforçado para dispor de material dedicado à doença de Alzheimer, especialmente por causa da revolução demográfica. A proporção de idosos aumentou de 9,1\%, em 1999, para 11,3\%, em 2009, no país, compondo hoje um contingente de aproximadamente 22 milhões de pessoas e superando a população de idosos de países europeus como a França, a Inglaterra e a Itália. Essa realidade torna-se mais expressiva quando se constata a relação diretamente proporcional que a DA mantém com aumento da idade 3 .

Assim, os países em desenvolvimento como o Brasil serão os mais impactados com essa transição, pois não estão preparados para lidar com o problema que sobrecarregará seus já claudicantes sistemas de saúde 1 . Esse também foi um ponto relevante nas publicações de Israel ${ }^{16}$ e do $\mid$ raân $^{11,19}$, onde a DA ainda se mostra obscura para cuidadores, o que exige maior número de investigações entre os especialistas desses países asiáticos. Muitos pontos nebulosos foram apresentados em artigos dos Estados Unidos ${ }^{17,18}$, onde a doença custa anualmente à sociedade cerca de US\$183 bilhões ${ }^{3}$. Apesar de tanto investimento em fatores que envolvem a DA (como tratamento, exames e cuidados), os cuidadores norte-americanos não se sentem à vontade diante dos problemas de comportamento e de comunicação dos idosos que têm a enfermidade.

Também ocupando grande parte da América do Norte como os Estados Unidos, o Canadá, que aparece no ranking dos países com um dos maiores índices de desenvolvimento humano (IDH), reconhece a necessidade de apoiar e orientar os cuidadores de idosos com Alzheimer, ao desenvolver e implementar um programa de intervenção psicoeducacional cujo objetivo é facilitar, para um parente de idoso com a doença, a transição para o papel de cuidador, logo após a família receber a notícia de diagnóstico da doença de Alzheimer $^{15}$.
Ainda em relação à procedência dos artigos selecionados, é importante reforçar o aparecimento dos países europeus: França ${ }^{12}$, Polônia ${ }^{14}$ e Inglaterra (Reino Unido) ${ }^{20}$. Na Europa, observa-se que pesquisadores têm dado maior ênfase ao estudo dos aspectos relacionados à importância da capacitação dos cuidadores, que relatam interesse em se informar sobre Alzheimer, a fim de melhorar a gestão de vida diária do idoso que tem a doença.

Dessa maneira, julga-se importante classificar os 12 artigos selecionados, nesta revisão de literatura, segundo abordagem: Conhecimento acerca do declínio cognitivo e funcional da doença de Alzheimer; Conhecimento acerca dos sintomas psicológicos e comportamentais da demência (SPCD); Conhecimento acerca do comprometimento da linguagem.

\section{Conhecimento acerca do declínio cognitivo e funcional da doença de Alzheimer}

No que se refere à DA, a carência de esclarecimentos norteadores atinge grande parcela de profissionais da área da saúde e cuidadores, que enfrentam - ao longo da evolução da doença - a dúvida do que fazer. Em se tratando de doença neurológica crônico-degenerativa, traz consigo dúvidas em relação ao manejo do doente, afetando aspectos de ordem pessoal, emocional, financeira e social do paciente e seus familiares ${ }^{22}$.

Para explorar as necessidades e as expectativas dos cuidadores familiares de idosos com DA em fase moderada que solicitaram assistência médica para seus parentes com a doença, Amieva et al. ${ }^{12}$ analisaram 645 cuidadores que faziam parte de um estudo randomizado multicêntrico. Os resultados demonstraram que $40 \%$ dos cuidadores relataram expectativas altas ou muito altas diante de necessidades relacionadas à informação sobre a doença (tratamento e prognóstico), como também diante de aprendizado, a fim de melhorar a gestão de vida diária do parente.

A desorientação quanto à patologia, que realmente atinge os cuidadores de idosos com DA, pode gerar um cuidado não específico, representado pela estagnação ou progressão insatisfatória do quadro clínico, causando sobrecarga daquele que desempenha o cuidado ${ }^{23}$.

Essa falta de orientação a respeito do que é a doença e sua evolução pode interferir na maneira como o cuidador planeja e executa as ações de cuidado, como mostrou o estudo de Lenardt et al. ${ }^{6}$, de caráter qualitativo, com o objetivo de revelar o sistema de conhecimento e de assistência de cuidadores familiares de idosos portadores da doença.

Os autores, então, concluíram que o conhecimento dos cuidadores sobre DA limita-se a informações fornecidas pela mídia, por folders distribuídos à população e pelo conhecimento do senso comum, o que contribui para cuidados pouco recomendáveis. Percebeu-se que o cuidado é desenvolvido de modo acrítico. Dessa maneira, fornecer 
informações é fundamental para desenvolver no cuidador a tomada de decisão no momento de prestar o cuidado ao idoso com DA.

A deficiência de orientação para o cuidado pode colocar em risco a saúde do idoso. No entanto, quando o cuidador é adequadamente instrumentalizado, torna-se capaz de enfrentar com maior segurança os desafios impostos pelo ato de cuidar ${ }^{24}$. Ainda assim, é visível a carência de suporte profissional e de redes de apoio com o intuito de minimizar o impacto na dinâmica familiar e na qualidade de vida do cuidador ${ }^{7}$.

O estudo de Cisek et al. ${ }^{14}$, desenvolvido com o objetivo de avaliar o nível de desenvoltura da família para cuidar de um parente com DA, observou que 67\% das famílias assistem o parente com a doença sem pertencer a grupo de apoio. Para esses familiares, adaptar-se à situação imposta pela enfermidade é difícil, embora a maioria deles não abra mão de dedicar parte da energia para o cuidado do paciente após o diagnóstico da doença.

Observa-se que programas educacionais são válidos e podem levar à maior confiança entre aqueles que exercem o cuidar. Extensa revisão na literatura, que agregou 380 artigos, feita pela American Academy of Neurology, sobre tratamentos não farmacológicos para a DA, mostrou que educação intensiva de longa duração e um programa de auxílio para cuidadores atrasaram a necessidade de internar o paciente em casa de repouso em 12 a 24 meses. Além disso, a educação dos cuidadores melhorou o nível de saúde do paciente, ainda que não tivesse impacto nos sintomas da doença ou em seus problemas de comportamento. A mesma pesquisa demonstrou que o treinamento especializado dos funcionários da casa de repouso pode reduzir significativamente o uso de antipsicóticos em pacientes com DA ${ }^{25}$. Dessa maneira, percebeu-se que a educação em saúde pode ser uma estratégia eficiente nesse tipo de atuação ${ }^{17}$.

Esse fato foi observado no estudo de Ducharme et al. ${ }^{15}$, que testou a eficácia de um programa psicoeducacional individual concebido para facilitar a transição de um indivíduo para o papel de cuidador após o diagnóstico da doença em um parente. Os resultados mostraram que, em comparação com o grupo de cuidadores usuais, os cuidadores do grupo experimental que receberam treinamento estavam mais confiantes em lidar com situações de cuidado, perceberam estar mais bem preparados para prestar cuidados, foram mais capazes de planejar as necessidades futuras de atenção ao seu parente com a doença e fizeram uso frequente das estratégias de enfrentamento de problemas.

Já o estudo de Werner ${ }^{16}$, concebido com a proposta de avaliar o nível de conhecimento da família sobre a DA e seus correlatos, mostrou que os cuidadores familiares pouco sabiam sobre itens relacionados a prevalência, a causas e a sintomas da doença. Assim, o autor concluiu que mais pesquisas sobre o conhecimento dos cuidadores sobre Alzheimer devem ser promovidas, a fim de criar ou estabelecer oportunidades para implementação de programas de intervenção, o que tende a permitir melhores tomadas de decisão dos cuidadores.

Nesse sentido, constata-se que a intervenção educativa pode favorecer o conhecimento e a ação dos cuidadores diante da pessoa com DA. A educação em saúde propicia também o aprendizado de novas formas de cuidar, ampliando as oportunidades para os cuidadores resgatarem o bem-estar físico e emocional ${ }^{24}$.

O estudo de Navab et al. ${ }^{11}$, cuja intenção foi compreender as experiências de cuidadores familiares de pessoas com DA, realizou entrevistas semiestruturadas baseadas na metodologia qualitativa com oito cuidadores. Os resultados enfatizaram a necessidade de proporcionar educação continuada e apoio para cuidadores familiares de pessoas com a doença. Com isso, os autores concluíram que os enfermeiros estão entre os profissionais da linha de frente para apoiar e fornecer informações para esses cuidadores.

Reconheceu-se esse panorama em estudo de Freitas et al. ${ }^{5}$, cujo objetivo foi descrever e analisar a convivência com o portador de Alzheimer sob a perspectiva do familiar cuidador. Observou-se que a DA é uma doença familiar e, para tanto, necessita de uma abordagem singular do enfermeiro, que deve assumir seu papel de educador e pesquisador e envolver-se diretamente na assistência prestada.

Com o objetivo de identificar e analisar a percepção do cuidador sobre os cuidados necessários prestados aos idosos com a doença, foi realizada outra pesquisa de caráter qualitativo, com entrevistas semiestruturadas com oito cuidadores. Os autores (Caldeira e Ribeiro) 4 perceberam que, pelo fato de os participantes do estudo não se sentirem bem diante do cuidar, é necessária uma assistência de enfermagem sistematizada de educação para os cuidadores de idosos com DA.

Outro estudo, de Yektatalab et al. ${ }^{19}$, também desenvolvido com base na metodologia qualitativa, teve como proposta explorar as percepções dos cuidadores iranianos de idosos com Alzheimer nas instituições de longa permanência de idosos, por meio de grupos focais e de entrevistas com 14 cuidadores. Os resultados mostraram que os participantes do estudo preocupam-se com o cuidado personalizado, o que exige planos de educação continuada para esses cuidadores. Essa orientação, segundo os autores, pode melhorar a qualidade do atendimento e a qualidade de vida do paciente.

\section{Conhecimento acerca dos sintomas psicológicos e comportamentais da demência}

Compreender onde estão as lacunas no conhecimento sobre DA pode favorecer a orientação de iniciativas de educação capazes de aumentar a conscientização sobre a doença e melhorar os serviços de apoio ${ }^{26}$. É importante também 
para os cuidadores conhecer os sintomas psicológicos e comportamentais da demência. Esses são os principais determinantes de sofrimento dos pacientes e da sobrecarga do cuidador ${ }^{27}$.

Em alguns estudos comunitários, avaliou-se a prevalência dos SPCD sistematicamente, como em publicação na qual foram encontrados sintomas neuropsiquiátricos em 75\% dos pacientes com demência, sendo as alterações mais comuns apatia (36\%), depressão (32\%) e agitação/agressividade $(30 \%)^{28}$.

O estudo qualitativo de Paton et al. ${ }^{20}$ foi especialmente desenvolvido com a intenção de verificar a compreensão de cuidadores acerca das causas dos sintomas comportamentais. Os resultados mostraram que a maioria dos participantes da pesquisa atribui os sintomas cognitivos, comportamentais e psicológicos da demência a outras causas não relacionadas à demência. Muitos acreditam que a pessoa com demência tem o controle sobre o seu comportamento e que vai voltar ao normal. Dessa maneira, os autores perceberam que informar sobre DA apenas não basta. É preciso oferecer uma educação continuada capaz de explorar as atribuições dos cuidadores diante dos sintomas comportamentais apresentados pelo idoso com a doença.

É fundamental orientar o familiar/cuidador sobre a importância de identificar a existência de algum fator associado ao aparecimento do sintoma ou comportamento alterado. A intervenção terapêutica para esses SPCD deve ser sempre iniciada por medidas não farmacológicas, que incluem adaptações ou modificações ambientais, instituição de rotinas específicas para os pacientes, orientações dirigidas aos cuidadores e familiares, além de programas de atividade física leve, emprego de música e terapia de luz ${ }^{9}$.

Para examinar as relações entre distúrbios de comportamento e o impacto no cuidado, a pesquisa de Robinson et al. ${ }^{17}$ foi feita com 30 cuidadores de idosos com Alzheimer. Chegou-se à conclusão de que esses participantes do estudo não se sentem à vontade diante dos problemas de comportamento dos idosos com a doença. Essas descobertas, segundo os autores, têm implicações para a implementação de programas de intervenção para cuidadores, que precisam de formação individualizada e específica para entender e gerenciar os sintomas comportamentais e a depressão em pessoas com Alzheimer.

Assim, percebe-se que a base da estratégia terapêutica para a DA inclui tratamento medicamentoso e intervenções não farmacológicas. Esse manejo clínico deve estar alicerçado em três pilares: retardar a evolução, controlar as alterações de comportamento e tratar os sintomas ${ }^{3}$.

\section{Conhecimento acerca do comprometimento da linguagem}

Cuidadores geralmente têm preocupações relacionadas aos domínios linguísticos alterados e às dificuldades comuni- cativas de quem convive com a DA. Diante do idoso com dificuldade em se comunicar, eles enfrentam um processo de intenso estresse e desgaste no relacionamento. Uma boa avaliação da linguagem e demais funções cognitivas possibilita a intervenção da linguagem, que visa à melhor qualidade de vida por meio de comunicação mais funcional e eficiente ${ }^{29}$.

Para descrever o conhecimento sobre comunicação na DA dos cônjuges cuidadores e determinar suas necessidades de aprendizado sobre estratégias de comunicação eficazes, o estudo desenvolvido por Williams ${ }^{18}$ mostrou que maridos e esposas dos idosos com a doença precisam compreender melhor o declínio da comunicação associado à DA. Conclui-se que enfermeiros podem desempenhar papel importante no apoio a casais, educando os cuidadores sobre distúrbios de comunicação, assim como sobre estratégias para vencê-los.

A literatura revela que estabelecer comunicação com o idoso com a doença não é fácil. A tarefa de manter esse diálogo deve abranger recursos como fala, escrita, gestos, entonação do discurso, atitudes e contato físico. O nível e a qualidade de comunicação dependerão do grau de comprometimento da memória e do raciocínio do paciente. Dependendo da fase da doença em que a pessoa com Alzheimer se encontra, determinadas características deverão nortear a estratégia a ser utilizada ${ }^{3}$.

O estudo de Roque et al. ${ }^{21}$, com objetivo de verificar a eficácia de um programa de orientação sobre estratégias comunicativas a cuidadores de idosos com DA moderada, observou a falta de consciência dos problemas de comunicação por parte dos cuidadores, que negavam dificuldades de comunicação com os dementes. Os pesquisadores também perceberam que os cuidadores tendem a atribuir pouca importância à comunicação diante das demais limitações geradas pela doença. Dessa maneira, conclui-se que programas educativos sobre o uso de estratégias comunicativas podem ser eficazes.

\section{CONCLUSÃO}

Por meio deste estudo, pode-se inferir que é extremamente necessário criar oportunidades para cuidadores familiares e profissionais conhecerem a doença para compreenderem o paciente, o processo evolutivo da DA e, consequentemente, as possibilidades de atuação (seguras e eficazes) nesse paciente. Na medida em que há investimento na formação e no processo de fornecimento de informação aos cuidadores, estima-se melhora na condução do caso, especialmente na qualidade de vida da população com a doença.

Neste artigo de revisão, esse cenário foi observado nos estudos selecionados, desenvolvidos em diversos países, o que mostra preocupação dos especialistas em todo o mundo com a ação dos cuidadores diante de um idoso com Al- 
zheimer. Assim, percebeu-se que a avaliação da qualidade do cuidado, os modelos educativos para cuidados em saúde e a qualidade de vida do idoso com Alzheimer são inquietações da comunidade científica mundialmente.

É notório que, diante do idoso com a doença, o cuidador pode apresentar dificuldades em compreender as mudanças e a progressão da DA, acreditando que as perdas cognitivas podem ser recuperadas com esforço. Essa falta de orientação a respeito da enfermidade pode interferir na maneira como o cuidador planeja e executa as ações de cuidado.

Dessa maneira, compreende-se que essa maneira de cuidar pode variar conforme a cultura de cada país, mas também de acordo com a educação em saúde, que propicia o aprendizado de novas formas de cuidar. Isso amplia oportunidades para os cuidadores resgatarem o próprio bem-estar físico e emocional, bem como favorece a autonomia e a tomada de decisão no momento de prestar cuidado ao idoso com Alzheimer.

\section{CONTRIBUIÇÕES INDIVIDUAIS}

Todas as autoras participaram da concepção do artigo, assim como da análise dos resultados, da discussão e da conclusão.

\section{CONFLITOS DE INTERESSE}

As autoras declaram não ter conflitos de interesse de qualquer natureza que possam ter influenciado na interpretação dos resultados aqui apresentados ou comprometido a integridade da pesquisa.

\section{REFERÊNCIAS}

1. Caixeta L. Evolução do conceito de doença de Alzheimer. In: Caixeta L (Org.). Doença de Alzheimer. Porto Alegre: Artmed; 2012.

2. Cordeiro Q, Vallada H. Bases genéticas da doença de Alzheimer. In: Caixeta L (Org.). Doença de Alzheimer. Porto Alegre: Artmed; 2012.

3. Sayeg N. Alzheimer - Diagnóstico e tratamento. São Caetano do Sul: Yendis; 2012.

4. Caldeira APS, Ribeiro RCHM. 0 enfrentamento do cuidador do idoso com Alzheimer. Ara Ciênc Saúde. 2004;11(2):100-4.

5. Freitas ICC, Paula KCC, Soares JL, Parente ACM. Convivendo com o portador de Alzheimer: perspectivas do familiar cuidador. Rev Bras Enferm [online]. 2008;61(4):508-13.

6. Lenardt MH, Silva SC, Willing MH, Seima MD. 0 idoso portador da doença de Alzheimer: 0 cuidado e o conhecimento do cuidador familiar. Rev Min Enferm. 2010;14(3):301-7.

7. Lopes DB, Caixeta L. 0 estresse dos cuidadores. In: Caixeta L (Org.). Doença de Alzheimer. Porto Alegre: Artmed; 2012
8. Resende MCF, Dias EC. Cuidadores de idosos: um novo/velho trabalho. Physis. 2008;18(4):785-800.

9. Caramelli P, Bottino CMC. Tratando os sintomas comportamentais e psicológicos da demência (SCPD). J Bras Psiquiatr. 2007;56(2):83-7.

10. Lopes L0, Cachioni M. Psychoeducational intervention for caregivers of elderly with dementia: a systematic review. J Bras Psiquiatr. 2012;61(4):252-61.

11. Navab E, Negarandeh R, Peyrovi H. Lived experiences of Iranian family member caregivers of persons with Alzheimer's disease: caring as 'captured in the whirlpool of time'. J Clin Nurs. 2012;21(7-8):1078-86.

12. Amieva H, Bouisson J, Dartigues JF, Dubois 0, Salamon R. Attentes et besoins des aidants de personnes souffrant de maladie d'Alzheimer. Rev Epidemiol Sante Publique. 2012;60:231-8.

13. Turato ER. Métodos qualitativos e quantitativos na área da saúde: definiç̧̃es, diferenças e seus objetos de pesquisa. Rev Saúde Pública. 2005;39(3):507-14.

14. Cisek M, Wilczek-Ruzyczka E, Gniadek A, Zdanowska A. Preparation the family to care of the patient with Alzheimer's disease. Rocz Akad Med Bialymst. 2005;50(1):116-8.

15. Ducharme FC, Lévesque LL, Lachance LM, Kergoat MJ, Legault AJ, Beaudet LM, et al. "Learning to become a family caregiver" efficacy of an intervention program for caregivers following diagnosis of dementia in a relative. Gerontologist. 2011;51(4):484-94.

16. Werner P. Correlates of family caregivers' knowledge about Alzheimer's disease. Int J Geriatr Psychiatry. 2001;16(1):32-8.

17. Robinson KM, Adkisson P, Weinrich S. Problem behaviour, caregiver reactions, and impact among caregivers of persons with Alzheimer's disease. J Adv Nurs. 2001;36(4):573-82.

18. Williams CL. What spouse caregivers know about communication in Alzheimer's disease: development of the AD communication knowledge test. Issues Ment Health Nurs. 2011;32(1):28-34.

19. Yektatalab SH, Kaveh MH, SharifF, Fallahi Khoshknab M, Petramfar P. Characteristics of care and caregivers of Alzheimer's patients in elderly care homes: a qualitative research. Iran Red Crescent Med J. 2012;14(5):294-9.

20. Paton J, Johnston K, Katona C, Livingston G. What causes problems in Alzheimer's disease: attributions by caregivers. A qualitative study. Int J Geriatr Psychiatry. 2004;19(6):527-32.

21. Roque FP, Ortiz KZ, Araújo MSC, Bertolucci PHF. Eficácia de treinamento de estratégias comunicativas a cuidadores de pacientes com demência. Pró-Fono R Atual Cient. 2009;21(3):225-30.

22. Poltroniere S, Cecchetto F, Souza EN. Doença de Alzheimer e demandas de cuidados: 0 que os enfermeiros sabem?. Rev Gaúcha Enferm. 2011;32(2):270-8.

23. Moraes EN, Santos RR. Demências irreversíveis. In: Moraes EN. Princípios básicos de geriatria e gerontologia. Belo Horizonte: Coopmed; 2008.

24. Martins JJ, Albuquerque GL, Nascimento ERP, Barra DCC, Souza WGA, Pacheco WNS. Necessidades de educação em saúde dos cuidadores de pessoas idosas no domićílio. Texto Contexto Enferm. 2007;16(2):254-62.

25. Doody R, Stevens J, Beck C, Dubinsky R, Kaye J, Gwyther L, et al. Practice parameter: management of dementia (an evidence-based review). Neurology. 2001;56(9):1154-66.

26. Carpenter BD, Zoller SM, Balsis S, Otilingam PG, Gatz M. Demographic and contextual factors related to knowledge about Alzheimer's disease. Am J Alzheimers Dis Other Demen. 2011;26(2):121-6.

27. Mckeith I, Cummings J. Behavioural changes and psychological symptoms in dementia disorders. Lancet Neurol. 2005;4(11):735-42.

28. Lyketsos CG, Lopez O, Jones B, Fitzpatrick AL, Breitner J, DeKosky S. Prevalence of neuropsychiatric symptoms in dementia and mild cognitive impairment: results from the cardiovascular health study. JAMA. 2002;288(12):1475-83.

29. Soares CD, Brandão L, Lacerda MC. Linguagem e discurso na doença de Alzheimer. In: Caixeta L (Org.). Doença de Alzheimer. Porto Alegre: Artmed; 2012. 